\title{
Benefits and risks of manual hyperinflation in intubated and mechanically ventilated intensive care unit patients: a systematic review
}

Frederique Paulus ${ }^{1 *}$, Jan M Binnekade ${ }^{1}$, Margreeth B Vroom ${ }^{1}$ and Marcus J Schultz ${ }^{1,2}$

\begin{abstract}
Introduction: Manual hyperinflation $(\mathrm{MH})$, a frequently applied maneuver in critically ill intubated and mechanically ventilated patients, is suggested to mimic a cough so that airway secretions are mobilized toward the larger airways, where they can easily be removed. As such, MH could prevent plugging of the airways.

Methods: We performed a search in the databases of Medline, Embase, and the Cochrane Library from January 1990 to April 2012. We systematically reviewed the literature on evidence for postulated benefits and risks of MH in critically ill intubated and mechanically ventilated patients.

Results: The search identified 50 articles, of which 19 were considered relevant. We included 13 interventional studies and six observational studies. The number of studies evaluating physiological effects of $\mathrm{MH}$ is limited. Trials differed too much to permit meta-analysis. It is uncertain whether $\mathrm{MH}$ was applied similarly in the retrieved studies. Finally, most studies are underpowered to show clinical benefit of $\mathrm{MH}$. Use of $\mathrm{MH}$ is associated with short-term improvements in lung compliance, oxygenation, and secretion clearance, without changes in outcomes. MH has been reported to be associated with short-term and probably clinically insignificant side effects, including decreases in cardiac output, alterations of heart rates, and increased central venous pressures.

Conclusions: Studies have failed to show that MH benefits critically ill intubated and mechanically ventilated patients. MH is infrequently associated with short-term side effects.
\end{abstract}

\section{Introduction}

Manual hyperinflation (MH), also known as "bagging" or "bag-squeezing" is a frequently used maneuver in critically ill intubated and mechanically ventilated patients $[1,2]$. With $\mathrm{MH}$, patients are disconnected from the mechanical ventilator, after which their lungs are temporarily ventilated with a manual ventilation bag. By applying a larger-than-normal volume at a low inspiratory flow followed by an inspiratory pause and expiration with a high expiratory flow, $\mathrm{MH}$ is suggested to mimic a normal cough. Propagation of airway secretions from the smaller toward the larger airways then allows for easy removal of airway secretions with airway suction. As such, $\mathrm{MH}$ could prevent airway plugging [3,4], and even promote alveolar recruitment [5].

\footnotetext{
*Correspondence: f.paulus@amc.uva.nl

'Department of Intensive Care Medicine, Academic Medical Center,

Meibergdreef 9, 1105 AZ, Amsterdam, The Netherlands

Full list of author information is available at the end of the article
}

It is far from certain whether MH truly benefits critically ill intubated and mechanically ventilated patients. In addition, disconnection of a critically ill patient from the ventilator could be seen as a rather unsafe intervention [6]. Because MH may cause short-term hyperinflation, one could even consider $\mathrm{MH}$ to be dangerous in hemodynamically unstable patients $[7,8]$. Also, $\mathrm{MH}$ could be disadvantageous in patients with respiratory failure. The airway pressures at the end of the $\mathrm{MH}$ maneuver are usually much lower than the applied level of positive end-expiratory pressure, which, in combination with airway suctioning, may promote atelectasis.

This systematic review aims to collect the evidence for the suggested benefits and risks of $\mathrm{MH}$ in critically ill intubated and mechanically ventilated patients. The main research questions were as follows. Does MH benefit critically ill intubated and mechanically ventilated patients with respect to pulmonary compliance, arterial oxygenation, and sputum clearance? Does MH have an effect on
C Biomed Central

(c) 2012 Paulus et al.; licensee BioMed Central Ltd. This is an open access article distributed under the terms of the Creative Commons Attribution License (http://creativecommons.org/licenses/by/2.0), which permits unrestricted use, distribution, and reproduction in any medium, provided the original work is properly cited. 
the duration of mechanical ventilation, length of stay in the intensive care unit, and incidence of pneumonia? What are reported side effects of $\mathrm{MH}$ ?

\section{The rationale behind manual hyperinflation}

Retained airway secretions may occlude the airways of intubated and mechanically ventilated patients, and, as such, cause atelectasis. This may impair oxygenation by increased intrapulmonary shunting and increase pulmonary vascular resistance. Large atelectasis may even promote development of lung injury [9]. The consequence of large atelectasis is a smaller lung available for ventilation, leading to the concept of "baby lung" ventilation [10]. Persistent presence of sputum in the airways may provide an ideal environment for colonizing organisms, finally resulting in pneumonia [11].

Frequent removal of sputum from the airways via tracheal suctioning is mandatory in critically ill intubated and mechanically ventilated patients. Under normal conditions, mucociliary transport clears the smaller airways of airway secretions. Secretions that are transported from the smaller airways into the bronchi and trachea then are removed by coughing. Critically ill patients, however, are frequently sedated and nursed in a supine position, potentially reducing mucociliary transport and promoting retention of airway secretions [12,13]. In addition, the cough reflex can be minimal or even absent in sedated critically ill patients, or they may lack force to cough efficiently. Furthermore, sputum may not be easily transported from the trachea into the translaryngeal tube or trachea cannula, and thus could remain in the larger airways. Unfortunately, with airway suctioning, only the trachea is cleared of secretions, as suction catheters cannot reach sputum in the bronchi and smaller airways. $\mathrm{MH}$, as originally described in the late 1960s, was designed to enhance clearance of airway secretions [14].

\section{Description of the MH technique}

To enhance the clearance of airway secretions, $\mathrm{MH}$ was supposed to include the application of a larger than normal volume (up to one and one half the size of tidal volumes delivered by the ventilator) at a low inspiratory flow (achieved by a slow compression of the ventilation bag), an inspiratory pause (to allow complete distribution of the inflated air among all the ventilated parts of the lung), and a high expiratory flow. In particular, this last element seems important and can be achieved by a complete and rapid release of the ventilation bag [15,16]. As such, MH could resemble a forceful cough, with which a forced and rapid exhalation follows a deep and slow inhalation.

It is suggested that the effectiveness of $\mathrm{MH}$ is dependent, at least in part, on the ratio between flows with inspiration and expiration $[3,4]$. With higher expiratory flows (that is, higher than inspiratory flows), sputum could propagate from distal to more proximal areas (that is, from the smaller airways toward the larger airways), where it can be easily removed through endotracheal suctioning $[17,18]$. It is also suggested that the use of an inspiratory pause maintains the pressure gradient for an appropriate length of time required to overcome the opening pressure of the alveoli [19].

\section{Materials and methods}

\section{Search methods for identification of manuscripts about $\mathrm{MH}$}

Two methods were used to identify relevant manuscripts in the medical literature. First, we performed a search in the databases of Medline, Embase, the Cochrane Library, the Cochrane Database of Systematic Reviews, and the Database of Abstracts on Reviews and Effectiveness (DARE) from January 1990 to April 2012 (Additional file 1). Second, reference lists of identified and selected manuscripts were reviewed to identify additional articles.

The following key words (MeSH and text words) were used: "critical care," "intensive care," "manual hyperinflation," "hyperinflation," "bagging," and "bag squeezing." In addition, we used the key words "hyperoxygenation," "physiotherapy," and "physical therapy." We excluded studies of mechanical hyperinflation by machines, like cough-assist devices. The initial search strategy was designed for maximal retrieval, with no limitation on the type of study design to be identified. We used no restriction on language.

\section{Study selection}

Two authors (FP and JB) independently reviewed the retrieved articles and abstracts, assessed the eligibility of each study, and resolved disagreement by consensus. Articles were selected if they reported original data from a clinical trial or an observational study. We restricted the selection of articles to those that reported on adult critically ill intubated and mechanically ventilated patients. The same authors made the final selection; we restricted the selection to articles that reported on relevant study end points, including pulmonary compliance, arterial oxygenation, sputum clearance, duration of mechanical ventilation, length of stay in the intensive care unit, and incidence of pneumonia, and only if the main objective concerned the evaluation of the $\mathrm{MH}$ procedure.

\section{Data-collection process}

We extracted data from the included studies by using a data-extraction sheet. We extracted the following data: characteristics of the studies (design, setting), participants, intervention characteristics ( $\mathrm{MH}$ technique), comparison intervention, and results of all relevant outcomes. 
Assessment of methodologic quality of individual studies Two reviewers (FP and JB) assessed the risk of bias of the interventional studies and used the categories randomization, random sequence generation allocation concealment, description of withdrawals and dropouts, the method of and use of intention-to-treat analysis, and standardization of important co-interventions.

\section{Synthesis of results}

The decision to combine studies in a quantitative analysis was made by assessing clinical heterogeneity (examining types of participants, interventions, and outcomes in each study).

\section{Results}

\section{Study selection}

The search (Figure 1) identified 50 articles, of which 19 were considered relevant (Tables 1, 2, and 3).

\section{Study characteristics}

We included 13 interventional studies (six randomized controlled trials of $\mathrm{MH}$ [5,20-24], two randomized crossover trials comparing $\mathrm{MH}$ with endotracheal suctioning [25,26], four randomized crossover trials comparing $\mathrm{MH}$ with hyperinflation by the mechanical ventilator [27-30], and one randomized crossover trial comparing two different manual-ventilation bags [31]), and six observational studies [7,8,32-35]. Physiological end points were respiratory mechanics [20-22,25,27-31],

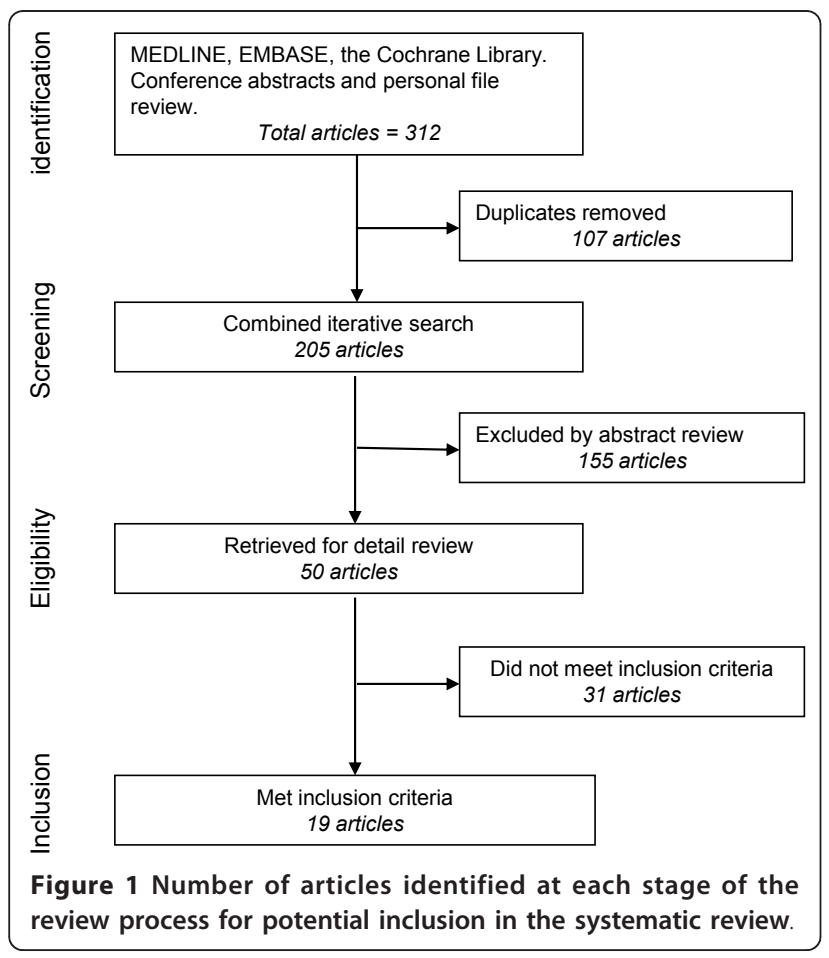

arterial oxygenation [5,20-24,26-28,30,31], and clearance of airway secretions [5,26,27,29-31]. Clinical end points included duration of mechanical ventilation [21,23], length of stay [21,23], and incidence of pneumonia [23]. Reported side effects included effects on heart rate and systemic blood pressure [7,8,20,26,32-34], cardiac output $[7,8,32,33,35]$, and pulmonary artery pressures and central venous pressures $[32,33,35]$.

\section{Risk of bias within studies}

The risk of bias among included studies is summarized in Table 4. Quality assessment revealed that five studies did not describe concealment of allocation. Only three studies clearly reported standardization of important cointerventions to prevent performance bias. Most studies did not report the use of intention-to-treat analysis. Description of the losses to follow-up was not included in three studies. All studies were open label, because blinding of the ICU clinicians was not feasible for these types of studies.

\section{Synthesis of results}

Studies varied widely in terms of patient populations, with dissimilar reasons for (acute and/or persistent) intubation and mechanical ventilation, $\mathrm{MH}$ intervention, and outcome measurements. Because of the substantial clinical heterogeneity, we focused on describing individual study results, rather than using a meta-analysis.

\section{Physiological end points}

The results of the physiological end points for the individual studies are summarized in Figures 2 and 3. We separated studies with an active control group (for example, hyperinflation by the ventilator) from studies comparing $\mathrm{MH}$ with standard care.

$\mathrm{MH}$ improved pulmonary compliance in postcardiac surgery patients [21,22], in patients with pneumonia [25], as well as in patients with large atelectasis [5]. Notably, $\mathrm{MH}$ did not improve pulmonary compliance in patients with acute lung injury [20]. With respect to changes in pulmonary compliance, $\mathrm{MH}$ was not superior to hyperinflation by the mechanical ventilator [27-30].

$\mathrm{MH}$ improved arterial oxygenation in studies of postcardiac surgery patients $[21,22]$. However, in another study of postcardiac surgery patients, this could not be confirmed [24], nor in studies of patients with atelectasis [5], nor in a study of brain-injury patients [23]. Notably, although no effect of $\mathrm{MH}$ on arterial oxygenation was found in a study of patients with acute lung injury [20], another study found $\mathrm{MH}$ to improve arterial oxygenation in patients with indirect acute lung injury but not in patients with direct acute lung injury [7,33]. $\mathrm{MH}$ was not superior to hyperinflation by the mechanical ventilator with respect to arterial oxygenation [27-30]. 
Table 1 Studies comparing manual hyperinflation (MH) with standard care

\begin{tabular}{|c|c|c|c|c|c|c|c|}
\hline $\begin{array}{l}\text { Author } \\
\text { [ref] }\end{array}$ & Year & Subjects & $N$ & Study design & Intervention & $\begin{array}{l}\text { Reported aspects of } \\
\text { the MH maneuver }\end{array}$ & Main results \\
\hline $\begin{array}{l}\text { Hodgson } \\
\text { et al. [26] }\end{array}$ & 2000 & $\begin{array}{l}\text { Unselected intensive } \\
\text { care unit patients }\end{array}$ & 18 & $\begin{array}{l}\text { Randomized } \\
\text { crossover trial }\end{array}$ & MH + endotracheal suction vs. standard care & $\begin{array}{l}\text { Volume: - } \\
\text { Inspiration speed: - } \\
\text { Pause: + } \\
\text { Expiration speed: } \pm\end{array}$ & $\begin{array}{l}\mathrm{MH} \text { increased pulmonary compliance but did not affect } \mathrm{P} / \mathrm{F} \text {; } \\
\mathrm{MH} \text { increased clearance of airway secretions }\end{array}$ \\
\hline $\begin{array}{l}\text { Patman et } \\
\text { al. [22] }\end{array}$ & 2000 & $\begin{array}{l}\text { Postcardiac surgery } \\
\text { patients }\end{array}$ & 94 & $\begin{array}{l}\text { Randomized } \\
\text { controlled trial }\end{array}$ & $\mathrm{MH}+$ endotracheal suction vs. standard care & $\begin{array}{l}\text { Volume: - } \\
\text { Inspiration speed: - } \\
\text { Pause: + } \\
\text { Expiration speed: - }\end{array}$ & MH increased pulmonary compliance and P/F \\
\hline $\begin{array}{l}\text { Barker et } \\
\text { al. [20] }\end{array}$ & 2002 & $\begin{array}{l}\text { Patients with acute } \\
\text { lung injury }\end{array}$ & 17 & $\begin{array}{l}\text { Randomized } \\
\text { controlled trial }\end{array}$ & $\begin{array}{l}\mathrm{MH}+\text { endotracheal suction + position } \\
\text { changes of patient vs. position changes only }\end{array}$ & $\begin{array}{l}\text { Volume: + } \\
\text { Inspiration speed: - } \\
\text { Pause : + } \\
\text { Expiration speed: - }\end{array}$ & MH affected neither pulmonary compliance nor P/F \\
\hline $\begin{array}{l}\text { Choi et al. } \\
\text { [25] }\end{array}$ & 2005 & $\begin{array}{l}\text { Patients with } \\
\text { pneumonia }\end{array}$ & 15 & $\begin{array}{l}\text { Randomized } \\
\text { crossover trial }\end{array}$ & $\mathrm{MH}+$ endotracheal suction vs. standard care & $\begin{array}{l}\text { Volume: } \pm \\
\text { Inspiration speed: - } \\
\text { Pause: }+ \\
\text { Expiration speed: + }\end{array}$ & MH improved pulmonary compliance \\
\hline $\begin{array}{l}\text { Maa et al. } \\
{[5]}\end{array}$ & 2006 & $\begin{array}{l}\text { Patients with } \\
\text { atelectasis }\end{array}$ & 23 & $\begin{array}{l}\text { Randomized } \\
\text { controlled trial }\end{array}$ & $\mathrm{MH}+$ endotracheal suction vs. standard care & $\begin{array}{l}\text { Volume: - } \\
\text { Inspiration speed: + } \\
\text { Pause: }+ \\
\text { Expiration speed: } \pm\end{array}$ & $\mathrm{MH}$ affected neither P/F nor clearance of airway secretions \\
\hline $\begin{array}{l}\text { Blattner et } \\
\text { al. [21] }\end{array}$ & 2008 & $\begin{array}{l}\text { Postcardiac surgery } \\
\text { patients }\end{array}$ & 55 & $\begin{array}{l}\text { Randomized } \\
\text { controlled trial }\end{array}$ & $\mathrm{MH}+$ endotracheal suction vs. standard care & $\begin{array}{l}\text { Volume: - } \\
\text { Inspiration speed: - } \\
\text { Pause: } \pm \\
\text { Expiration speed: - }\end{array}$ & $\begin{array}{l}\mathrm{MH} \text { improved pulmonary compliance and } \mathrm{PaO}_{2} \text { and } \\
\text { reduced duration of MV }\end{array}$ \\
\hline $\begin{array}{l}\text { Patman et } \\
\text { al. [23] }\end{array}$ & 2009 & Brain-injury patients & 144 & $\begin{array}{l}\text { Randomized } \\
\text { controlled trial }\end{array}$ & $\begin{array}{l}\mathrm{MH}+\text { endotracheal suction and side lying vs. } \\
\text { standard care }\end{array}$ & $\begin{array}{l}\text { Volume: } \pm \\
\text { Inspiration speed: } \pm \\
\text { Pause: }+ \\
\text { Expiration speed: } \pm\end{array}$ & $\begin{array}{l}\mathrm{MH} \text { affected neither duration of MV and length of stay in } \\
\text { intensive care unit, nor the incidence of pneumonia }\end{array}$ \\
\hline $\begin{array}{l}\text { Paulus et } \\
\text { al. [24] }\end{array}$ & 2011 & $\begin{array}{l}\text { Postcardiac surgery } \\
\text { patients }\end{array}$ & 100 & $\begin{array}{l}\text { Randomized } \\
\text { controlled trial }\end{array}$ & $\mathrm{MH}+$ endotracheal suction vs. standard care & $\begin{array}{l}\text { Volume: }+ \\
\text { Inspiration speed: + } \\
\text { Pause: }+ \\
\text { Expiration speed: + }\end{array}$ & MH did not affect pulse-oximeter oxygen saturation \\
\hline
\end{tabular}

Reported aspects of the MH maneuver: volume, whether larger than normal breaths were used; inspiration speed, whether a low inspiratory flow was used; pause, whether pauses were used; expiration speed, whether rapid expiratory flows were used (+, mentioned; -, not mentioned; \pm , uncertain) 
Table 2 Studies comparing manual hyperinflation (MH) with other strategies

\begin{tabular}{|c|c|c|c|c|c|c|c|}
\hline $\begin{array}{l}\text { Author } \\
\text { [ref] }\end{array}$ & Year & Subjects & $N$ & Study design & Intervention & $\begin{array}{l}\text { Reported aspects } \\
\text { of the } \mathrm{MH} \\
\text { maneuver }\end{array}$ & Main results \\
\hline $\begin{array}{l}\text { Berney et } \\
\text { al. [29] }\end{array}$ & 2002 & $\begin{array}{l}\text { Unselected } \\
\text { intensive care unit } \\
\text { patients }\end{array}$ & 20 & $\begin{array}{l}\text { Randomized } \\
\text { crossover trial }\end{array}$ & $\begin{array}{l}\text { MH vs. hyperinflation } \\
\text { by the mechanical } \\
\text { ventilator }\end{array}$ & $\begin{array}{l}\text { Volume: } \pm \\
\text { Inspiration speed: }+ \\
\text { Pause: }+ \\
\text { Expiration speed: } \pm\end{array}$ & $\begin{array}{l}\text { Both techniques improved pulmonary compliance equally; no affects on clearance of } \\
\text { airway secretions }\end{array}$ \\
\hline $\begin{array}{l}\text { Paratz et } \\
\text { al. [33] }\end{array}$ & 2002 & $\begin{array}{l}\text { Patients with } \\
\text { acute lung injury }\end{array}$ & 16 & $\begin{array}{l}\text { Prospective } \\
\text { observational } \\
\text { study }\end{array}$ & $\mathrm{MH}$ & $\begin{array}{l}\text { Volume: + } \\
\text { Inspiration speed: + } \\
\text { Pause: }+ \\
\text { Expiration speed: + }\end{array}$ & $\begin{array}{l}\text { MH increased pulmonary compliance; } P / F \text { increased in patients with an extrapulmonary } \\
\text { cause of acute lung injury, whereas it decreased in patients with a pulmonary cause of } \\
\text { acute lung injury }\end{array}$ \\
\hline $\begin{array}{l}\text { Savian et } \\
\text { al. [30] }\end{array}$ & 2006 & $\begin{array}{l}\text { Unselected } \\
\text { intensive care unit } \\
\text { patients }\end{array}$ & 14 & $\begin{array}{l}\text { Randomized } \\
\text { crossover trial }\end{array}$ & $\begin{array}{l}\text { MH vs. hyperinflation } \\
\text { by the mechanical } \\
\text { ventilator }\end{array}$ & $\begin{array}{l}\text { Volume: - } \\
\text { Inspiration speed: + } \\
\text { Pause: }+ \\
\text { Expiration speed: + }\end{array}$ & $\mathrm{MH}$ affected neither pulmonary compliance and $\mathrm{PaO}_{2}$, nor clearance of airway secretions \\
\hline $\begin{array}{l}\text { Hodgson } \\
\text { et al. [31] }\end{array}$ & 2007 & $\begin{array}{l}\text { Unselected } \\
\text { intensive care unit } \\
\text { patients }\end{array}$ & 20 & $\begin{array}{l}\text { Randomized } \\
\text { crossover trial }\end{array}$ & $\begin{array}{l}\text { MH with two different } \\
\text { devices for } \\
\text { hyperinflation }\end{array}$ & $\begin{array}{l}\text { Volume: } \pm \\
\text { Inspiration speed: - } \\
\text { Pause: }+ \\
\text { Expiration speed: } \pm\end{array}$ & $\begin{array}{l}\text { MH affect neither pulmonary compliance nor P/F; clearance of airway secretions differed } \\
\text { between two devices }\end{array}$ \\
\hline $\begin{array}{l}\text { Ahmed et } \\
\text { al. [28] }\end{array}$ & 2010 & $\begin{array}{l}\text { Postcardiac } \\
\text { surgery patients }\end{array}$ & 30 & $\begin{array}{l}\text { Randomized } \\
\text { controlled trial }\end{array}$ & $\begin{array}{l}\text { MH vs. hyperinflation } \\
\text { by the mechanical } \\
\text { ventilator }\end{array}$ & $\begin{array}{l}\text { Volume: } \pm \\
\text { Inspiration speed: }+ \\
\text { Pause: }+ \\
\text { Expiration speed: + }\end{array}$ & $\begin{array}{l}\text { Both techniques improved P/F equally; both techniques did not affect pulmonary } \\
\text { compliance }\end{array}$ \\
\hline $\begin{array}{l}\text { Dennis et } \\
\text { al. [27] }\end{array}$ & 2012 & $\begin{array}{l}\text { Patients with } \\
\text { atelectasis }\end{array}$ & 46 & $\begin{array}{l}\text { Randomized } \\
\text { cross-over trial }\end{array}$ & $\begin{array}{l}\text { MH vs. hyperinflation } \\
\text { by the mechanical } \\
\text { ventilator }\end{array}$ & $\begin{array}{l}\text { Volume: } \pm \\
\text { Inspiration speed: - } \\
\text { Pause: }+ \\
\text { Expiration speed: - }\end{array}$ & $\begin{array}{l}\text { Clearance of airway secretions did not differ between the two techniques; both techniques } \\
\text { did not affect pulmonary compliance; P/F increased after VH but it decreased after } \mathrm{MH}\end{array}$ \\
\hline
\end{tabular}

Reported aspects of the MH maneuver: volume, whether larger than normal breaths were used; inspiration speed, whether a low inspiratory flow was used; pause, whether pauses were used; expiration speed, whether rapid expiratory flows were used $(+$, mentioned; - , not mentioned; \pm , uncertain). 
Table 3 Side effects of manual hyperinflation (MH)

\begin{tabular}{|c|c|c|c|c|c|c|c|}
\hline $\begin{array}{l}\text { Author } \\
\text { [ref] }\end{array}$ & Year & Subjects & $N$ & Study design & Intervention & $\begin{array}{l}\text { Reported aspects } \\
\text { of the } \mathrm{MH} \\
\text { maneuver }\end{array}$ & Main results \\
\hline $\begin{array}{l}\text { Singer et } \\
\text { al. [8] }\end{array}$ & 1994 & $\begin{array}{l}\text { Unselected } \\
\text { intensive care unit } \\
\text { patients }\end{array}$ & 18 & $\begin{array}{l}\text { Prospective } \\
\text { observational } \\
\text { study }\end{array}$ & $\begin{array}{l}\text { Measurements of hemodynamic parameters } \\
\text { before and after MH }\end{array}$ & $\begin{array}{l}\text { Volume: + } \\
\text { Inspiration speed:- } \\
\text { Pause: - } \\
\text { Expiration speed: - }\end{array}$ & $\begin{array}{l}\text { MH decreased cardiac output; MH did not affect heart rate or } \\
\text { systemic blood pressure }\end{array}$ \\
\hline $\begin{array}{l}\text { Jellema } \\
\text { et al. [32] }\end{array}$ & 2000 & $\begin{array}{l}\text { Patients with septic } \\
\text { shock }\end{array}$ & 13 & $\begin{array}{l}\text { Prospective } \\
\text { observational } \\
\text { study }\end{array}$ & $\begin{array}{l}\text { Measurements of hemodynamic parameters } \\
\text { before and after } \mathrm{MH}\end{array}$ & $\begin{array}{l}\text { Volume: } \pm \\
\text { Inspiration speed: - } \\
\text { Pause: + } \\
\text { Expiration speed: + }\end{array}$ & $\begin{array}{l}\text { MH did not affect cardiac output, heart rate, systemic blood } \\
\text { pressure, or central venous pressure }\end{array}$ \\
\hline $\begin{array}{l}\text { Paratz et } \\
\text { al. [33] }\end{array}$ & 2002 & Patients with ALI & 16 & $\begin{array}{l}\text { Prospective } \\
\text { observational } \\
\text { study }\end{array}$ & $\begin{array}{l}\text { Measurements of hemodynamic and } \\
\text { respiratory parameters before and after } \mathrm{MH}\end{array}$ & $\begin{array}{l}\text { Volume: + } \\
\text { Inspiration speed: + } \\
\text { Pause: + } \\
\text { Expiration speed: + }\end{array}$ & $\begin{array}{l}\text { MH did not affect cardiac output, heart rate, systemic blood } \\
\text { pressure, or central venous pressure }\end{array}$ \\
\hline $\begin{array}{l}\text { Paratz et } \\
\text { al. [7] }\end{array}$ & 2006 & Patients in shock & 7 & $\begin{array}{l}\text { Prospective } \\
\text { observational } \\
\text { study }\end{array}$ & $\begin{array}{l}\text { Measurements of hemodynamic parameters } \\
\text { and plasma catecholamines before and after } \\
\mathrm{MH}\end{array}$ & $\begin{array}{l}\text { Volume: + } \\
\text { Inspiration speed: + } \\
\text { Pause: }+ \\
\text { Expiration speed: + }\end{array}$ & $\begin{array}{l}\text { MH decreased cardiac output, and increased systemic vascular } \\
\text { resistance, and diastolic blood pressure }\end{array}$ \\
\hline $\begin{array}{l}\text { Hodgson } \\
\text { et al. [26] }\end{array}$ & 2000 & $\begin{array}{l}\text { Unselected } \\
\text { intensive care unit } \\
\text { patients }\end{array}$ & 18 & $\begin{array}{l}\text { Randomized } \\
\text { crossover trial }\end{array}$ & $\mathrm{MH}+$ endotracheal suction vs. standard care & $\begin{array}{l}\text { Volume: - } \\
\text { Inspiration speed: - } \\
\text { Pause: + } \\
\text { Expiration speed: } \pm\end{array}$ & MH did not affect heart rate or systemic blood pressure \\
\hline $\begin{array}{l}\text { Barker et } \\
\text { al. [20] }\end{array}$ & 2002 & Patients with ALI & 18 & $\begin{array}{l}\text { Randomized } \\
\text { controlled trial }\end{array}$ & $\begin{array}{l}\mathrm{MH}+\text { endotracheal suction vs. position } \\
\text { changes of patient }\end{array}$ & $\begin{array}{l}\text { Volume: + } \\
\text { Inspiration speed: - } \\
\text { Pause : + } \\
\text { Expiration speed: - }\end{array}$ & $\begin{array}{l}\text { MH increased heart rate and systemic blood pressure; MH did } \\
\text { not affect pulmonary artery wedge pressure }\end{array}$ \\
\hline $\begin{array}{l}\text { Paulus et } \\
\text { al. [34] }\end{array}$ & 2010 & $\begin{array}{l}\text { Unselected } \\
\text { intensive care unit } \\
\text { patients }\end{array}$ & 74 & $\begin{array}{l}\text { Prospective } \\
\text { observational } \\
\text { study }\end{array}$ & $\begin{array}{l}\text { Measurements of hemodynamic parameters } \\
\text { before and after } \mathrm{MH}\end{array}$ & $\begin{array}{l}\text { Volume: + } \\
\text { Inspiration speed: + } \\
\text { Pause: + } \\
\text { Expiration speed: + }\end{array}$ & $\begin{array}{l}\mathrm{MH} \text { did not affect systemic blood pressure or peripheral } \\
\text { oxygen saturation; } \mathrm{MH} \text { increased heart rate and end-tidal } \mathrm{CO}_{2} \\
\text { levels }\end{array}$ \\
\hline $\begin{array}{l}\text { Patman } \\
\text { et al. [35] }\end{array}$ & 1998 & $\begin{array}{l}\text { Postcardiac surgery } \\
\text { patients }\end{array}$ & 30 & $\begin{array}{l}\text { Prospective } \\
\text { observational } \\
\text { study }\end{array}$ & $\begin{array}{l}\text { Measurements of hemodynamic parameters } \\
\text { before and after } \mathrm{MH}\end{array}$ & $\begin{array}{l}\text { Volume: - } \\
\text { Inspiration speed: - } \\
\text { Pause: } \pm \\
\text { Expiration speed: - }\end{array}$ & $\begin{array}{l}\text { MH increased central venous pressures and decreased heart } \\
\text { rate; MH did not affect cardiac output }\end{array}$ \\
\hline
\end{tabular}

whether rapid expiratory flow was used $(+$, mentioned; - , not mentioned; \pm , uncertain). 
Table 4 Summary of risk of bias assessment of the interventional studies

\begin{tabular}{llllll}
\hline Author [ref] & $\begin{array}{l}\text { Random sequence } \\
\text { generation }\end{array}$ & $\begin{array}{l}\text { Allocation } \\
\text { concealment }\end{array}$ & $\begin{array}{l}\text { Standardization of co- } \\
\text { interventions }\end{array}$ & $\begin{array}{l}\text { Intention-to-treat } \\
\text { analysis }\end{array}$ & $\begin{array}{l}\text { Description of losses to } \\
\text { follow-up }\end{array}$ \\
\hline Hodgson et al. [26] & + & + & + & - & + \\
Patman et al. [22] & + & + & \pm & - & + \\
Barker et al. [20] & + & + & \pm & - & - \\
Choi et al. [25] & + & - & + & + & + \\
Maa et al. [5] & \pm & \pm & + & + & + \\
Blattner et al. [21] & + & + & + & + & + \\
Patman et al. [23] & + & - & - & - & + \\
Paulus et al. [24] & + & + & - & - & + \\
Berney et al. [29] & + & + & \pm & + & + \\
Savian et al. [30] & + & - & \pm & - & + \\
Hodgson et al. [31] & + & \pm & \pm & - & + \\
Ahmed et al. [28] & + & - & \pm & & + \\
Dennis et al. [27] & + & + & + & - & + \\
\hline
\end{tabular}

+ , mentioned; -, not mentioned; \pm , uncertain.

Although studies of unselected ICU patients found MH to improve sputum clearance [26,31], this was not found in a study of patients with atelectasis [5]. Similar volumes of pulmonary secretions were mobilized with $\mathrm{MH}$ compared with hyperinflation by the mechanical ventilator $[27,29]$.

\section{Clinical end points}

$\mathrm{MH}$ was found to shorten the duration of mechanical ventilation in a study of postcardiac surgery patients [21]. This, however, was not confirmed in a study of brain-injury patients [23]. MH did not reduce length of stay in the ICU or hospital, in either postcardiac surgery patients [21] or brain-injury patients [23]. In addition, $\mathrm{MH}$ did not reduce the incidence of pneumonia in brain-injury patients [23].

\section{Side effects}

The results of the side effects are summarized in Table 3. The majority of studies found $\mathrm{MH}$ not to affect systemic blood pressure and heart rate $[7,8,26,32,33]$. One study of patients with acute lung injury [20] and one observational study of unselected ICU patients [34] showed MH to increase heart rate and blood pressure, whereas another study in unselected patients showed $\mathrm{MH}$ to decrease heart rate [35]. MH was associated with a short-lived $10 \%$ to $15 \%$ decrease in cardiac output in patients with septic shock as well as in unselected ICU patients $[7,8]$. Conversely, this was not found in observational studies of patients with septic shock, patients with acute lung injury, and patients after cardiac surgery $[32,33,35]$. MH was associated with increased central venous pressures in one observational study of patients after cardiac surgery [35]. Side effects were frequently considered clinically irrelevant $[7,8,20,34]$.

\section{Discussion}

$\mathrm{MH}$ is suggested to mimic a cough so that airway secretions are mobilized from the smaller airways toward the larger airways, where they can easily be removed. As such, MH could benefit critically ill intubated and mechanically ventilated patients. We reviewed studies of diverse intensive care unit populations investigating the potential beneficial effects and side effects of $\mathrm{MH}$. Most investigations consistently showed $\mathrm{MH}$ to be feasible and safe. $\mathrm{MH}$ improved pulmonary compliance, arterial oxygenation, and clearance of airway secretions, albeit not in all investigations. $\mathrm{MH}$ inconsistently affected clinical outcome. Side effects of MH seemed relatively infrequent.

Apart from the possibility that MH may indeed not benefit critically ill intubated and mechanically ventilated patients, studies simply may have been underpowered to detect any beneficial effect of $\mathrm{MH}$, such as duration of mechanical ventilation, length of stay in the intensive care unit, and prevention of ventilator-associated pneumonia.

Studies in this review were heterogeneous in regard to patient populations, $\mathrm{MH}$ intervention, and outcome measurements and could not be combined in a metaanalysis. Only a few studies compared $\mathrm{MH}$ with standard care. In some studies, MH was compared with another strategy (for example, hyperinflation by the ventilator). Two of the retrieved studies reported the use of position changes in conjunction with $\mathrm{MH}$. Multiple other strategies could have been used in conjunction with $\mathrm{MH}$ in other studies, such as postural drainage, vibrations, and manually assisted cough. This, however, was not clearly reported. In addition, most studies included in this review had methodologic flaws, which may have resulted in bias. 


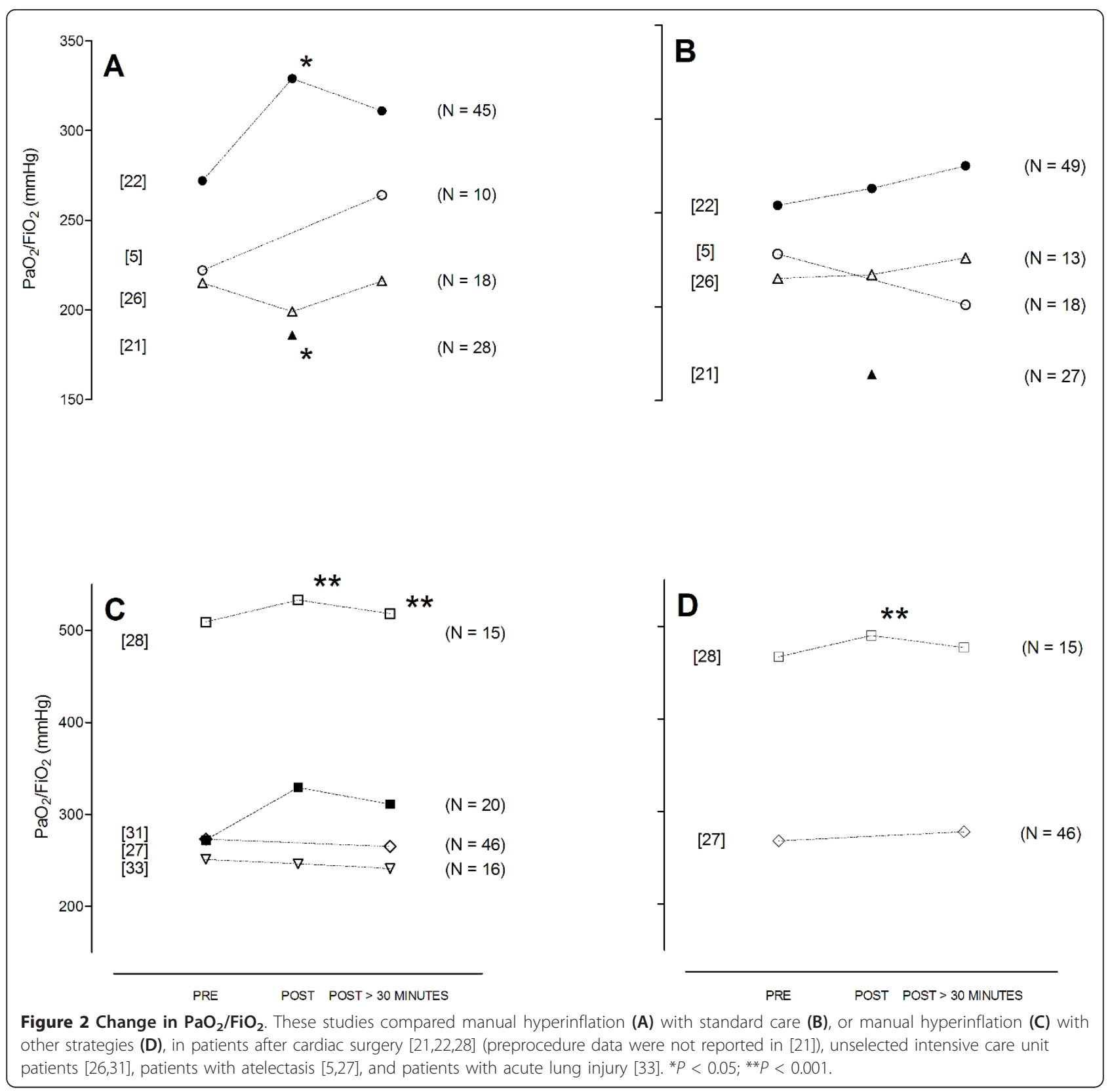

Better evidence to support the use of $\mathrm{MH}$ is required. Therefore, appropriately powered, well-designed, randomized controlled trials evaluating the effect of $\mathrm{MH}$ should be conducted. The focus of these studies should be on clinical end points, including, but not restricted to, duration of mechanical ventilation or ventilator-free days, length of stay in the ICU, and incidence of ventilator-associated pneumonia.

It is difficult to give clear recommendations on how to perform MH maneuvers. Most reports did not adequately describe how MH actually was performed. Monitoring airway pressures is feasible, but may not have priority. Notably, use of larger than normal breaths is associated with higher inspiratory airway pressures, up to $40 \pm 8 \mathrm{~cm} \mathrm{H}_{2} \mathrm{O}[36,37]$. Also, almost without exception expiratory airway pressures are low, as low as 2.1 [1.4 to 3.1] $\mathrm{cm} \mathrm{H}_{2} \mathrm{O}$ [37]. The most important aspect for $\mathrm{MH}$ to be effective may be the high expiratory flow, which can be achieved by rapid and complete release of the ventilation bag [16]. Outside the importance of conducting future studies that comprehensively describe the $\mathrm{MH}$ technique used, additional studies could establish how the different components of the technique are of influence on the therapeutic aims of $\mathrm{MH}$. 


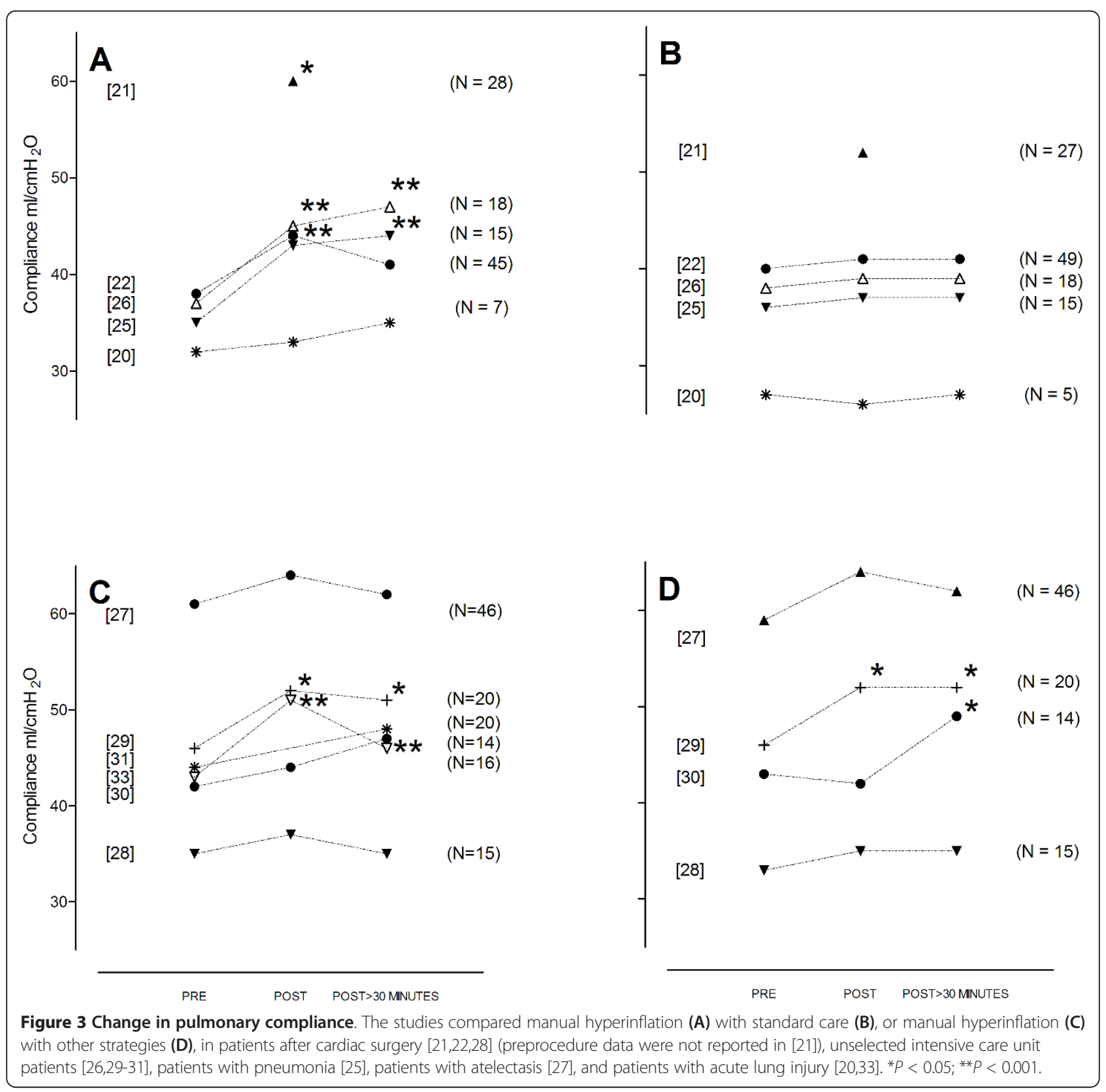

Unfortunately, $\mathrm{MH}$ is frequently referred to as a maneuver to recruit lung tissue. We would like to emphasize that $\mathrm{MH}$ was originally designed to mimic a forceful cough. The most important principle for $\mathrm{MH}$ to be effective may be the high expiratory flow. Consequently, airway pressures at the end of each $\mathrm{MH}$ cycle are low, which may very well promote derecruitment. With the performance of $\mathrm{MH}$, the necessity exists to disconnect the patient from the mechanical ventilator. Breaking the ventilatory circuit may lead to airway contamination and eventually to ventilator-associated pneumonia. Breaking the ventilatory circuit may also lead to significant airway pressure decreases and promote lung derecruitment.
Finally, the delivery of larger than normal tidal volumes with $\mathrm{MH}$, even for a very short time, may cause overinflation. Although scientific evidence for the existence of these potential side effects is lacking, they may very well limit adoption of $\mathrm{MH}$. Derecruitment and overinflation could be harmful, especially in patients with acute lung injury, or its more severe form, acute respiratory distress syndrome. It is imaginable that derecruitment and overinflation affect the lungs differently among diverse populations of ICU patients. We suggest that MH may better recruit lung tissue in patients with easy-to-recruit lungs (for example, patients after cardiac surgery or patients with indirect lung injury) than in patients with 
less compliant lungs (for example, patients with direct lung injury). The dissimilar findings of investigations reviewed here are in line with this suggestion

Future studies should include patients with different pulmonary conditions and should address overinflation and derecruitment with $\mathrm{MH}$. It may be necessary to add recruitment maneuvers to $\mathrm{MH}$, but as far as we know, this has not been the subject of clinical studies.

The retrieved studies reported side effects of $\mathrm{MH}$ relatively infrequently, and most of the reported side effects were minor. Because most if not all studies were not specifically designed to detect side effects of $\mathrm{MH}$, absence of reported side effects may not mean that the procedure is necessarily safe.

In a study in which patients showed a small decrease in cardiac output, it was hypothesized that this was caused by a decrease in the cardiac preload [8]. A decrease in cardiac output could therefore depend on the expertise and level of training of ICU nurses and/or respiratory therapists: a (too) large increase of the intrathoracic volume due to a (too) large tidal volume with $\mathrm{MH}$ may impede venous return [8,32]. It has been suggested that $\mathrm{MH}$, when performed under controlled conditions and/or performed by experienced and trained ICU nurses, respiratory therapists, and/or intensivists, has negligible effects on cardiac output [32-34].

Given the paucity of data, one might simply recommend abandoning $\mathrm{MH}$ as a relic of good intentions with little scientific evidence, but with potential harm. However, absence of evidence does not necessarily mean evidence of absence. A pathophysiological rationale exists for $\mathrm{MH}$ as a secretion-clearance technique that should be tested in clinical trials. From this review of studies, we conclude that until now, no adequately powered studies tested the hypothesis that MH benefits intubated and mechanically ventilated patients. The same also applies for the potential adverse events of $\mathrm{MH}$.

Limitations exist in the way we conducted our review. First, two of the authors are ICU clinicians and frequent users of the $\mathrm{MH}$ procedure and may be potentially biased. Second, electronic and hand searches do not completely reflect the extent of research outcomes; for example, studies presented at congresses are more likely to contain negative reports than are studies reported in the literature. Furthermore, many studies not published in English may not be included in the most commonly used searches.

\section{Conclusions}

$\mathrm{MH}$ is associated with short-term beneficial effects on lung compliance, oxygenation, and airway clearance in intubated and mechanically ventilated patients. $\mathrm{MH}$ is inconsistently associated with clinical benefit. $\mathrm{MH}$ only infrequently has been associated with side effects. It should be noted, though, that the majority of published studies were not designed to detect potential adverse events like derecruitment. Appropriately powered and methodologically sound studies of $\mathrm{MH}$ are needed before recommendations can be made for routine use of $\mathrm{MH}$.

\section{Key messages}

- Manual hyperinflation is a frequently used maneuver that intends to mimic a forceful cough in critically ill intubated and mechanically ventilated patients.

- Manual hyperinflation may improve pulmonary compliance, arterial oxygenation, and clearance of airway secretions.

- As such, manual hyperinflation may benefit intubated and mechanically ventilated critically ill patients.

- Side effects of manual hyperinflation seem relatively infrequent; however, most studies did not seem to be designed to detect potential adverse effects like derecruitment.

- Better evidence to support use of manual hyperinflation is required.

\section{Additional material}

Additional file 1: Search method. A summary of search strategy and search terms for the identification of articles with $\mathrm{MH}$.

\section{Abbreviations}

ICU: intensive care unit; MH: manual hyperinflation; MV: mechanical ventilation; P/F: ratio of partial pressure of arterial oxygen to fraction of inspired oxygen.

\section{Author details}

${ }^{1}$ Department of Intensive Care Medicine, Academic Medical Center, Meibergdreef 9, 1105 AZ, Amsterdam, The Netherlands. 'Laboratory for Experimental Intensive Care and Anesthesiology (L.E.I.C.A), Academic Medical Center, Meibergdreef 9, 1105 AZ, Amsterdam, The Netherlands.

\section{Authors' contributions}

FP and JMB performed the literature search and selected the relevant articles for inclusion independently. FP and JMB reviewed the selected articles. FP and JMB wrote the initial draft of the manuscript. MJS contributed to the interpretation of the results and drafting of the manuscript. MBV and MJS critically revised the manuscript. All authors read and approved the manuscript for publication.

\section{Competing interests}

The authors declare that they have no competing interests.

Received: 24 November 2011 Revised: 15 July 2012 Accepted: 3 August 2012 Published: 3 August 2012

\section{References}

1. Hodgson C, Carroll S, Denehy L: A survey of manual hyperinflation in Australian hospitals. Aust J Physiother 1999, 45:185-193.

2. Paulus F, Binnekade JM, Middelhoek P, Schultz MJ, Vroom MB: Manual hyperinflation of intubated and mechanically ventilated patients in 
Dutch intensive care units: a survey into current practice and knowledge. Intensive Crit Care Nurs 2009, 25:199-207.

3. Jones AY: Secretion movement during manual lung inflation and mechanical ventilation. Respir Physiol Neurobiol 2002, 132:321-327.

4. Kim CS, Iglesias AJ, Sackner MA: Mucus clearance by two-phase gas-liquid flow mechanism: asymmetric periodic flow model. J Appl Physiol 1987, 62:959-971.

5. Maa SH, Hung TJ, Hsu KH, Hsieh Yl, Wang KY, Wang CH, Lin HC: Manual hyperinflation improves alveolar recruitment in difficult-to-wean patients. Chest 2005, 128:2714-2721.

6. Lu Q, Capderou A, Cluzel P, Mourgeon E, Abdennour L, Law-Koune JD, Straus C, Grenier P, Zelter M, Rouby JJ: A computed tomographic scan assessment of endotracheal suctioning-induced bronchoconstriction in ventilated sheep. Am J Respir Crit Care Med 2000, 162:1898-1904.

7. Paratz J, Lipman J: Manual hyperinflation causes norepinephrine release. Heart Lung 2006, 35:262-268.

8. Singer M, Vermaat J, Hall G, Latter G, Patel M: Hemodynamic effects of manual hyperinflation in critically ill mechanically ventilated patients. Chest 1994, 106:1182-1187.

9. Duggan M, Kavanagh BP: Pulmonary atelectasis: a pathogenic perioperative entity. Anesthesiology 2005, 102:838-854.

10. Gattinoni L, Pesenti A: The concept of "baby lung". Intensive Care Med 2005, 31:776-784.

11. Fahy JV, Dickey BF: Airway mucus function and dysfunction. N Engl J Med 2010, 363:2233-2247.

12. Dolovich M, Rushbrook J, Churchill E, Mazza M, Powles AC: Effect of continuous lateral rotational therapy on lung mucus transport in mechanically ventilated patients. J Crit Care 1998, 13:119-125.

13. Marik PE, Fink MP: One good turn deserves another! Crit Care Med 2002, 30:2146-2148

14. Clement AJ, Hubsch SK: Chest physiotherapy by the 'bag squeezing' method: a guide to technique. Physiotherapy 1968, 54:355-359.

15. Maxwell $\amalg$, Ellis ER: The effect of circuit type, volume delivered and "rapid release" on flow rates during manual hyperinflation. Aust $J$ Physiother 2003, 49:31-38.

16. Paulus F, Binnekade JM, Middelhoek P, Vroom MB, Schultz MJ: Feedback and education improve manual hyperinflation maneuvers. Am J Respir Crit Care Med 2009, 179:A2306.

17. Ntoumenopoulos G: Mucus on the move: embed it or expel it: the patient, the clinician, and now the ventilator. Respir Care 2008, 53:1276-1279

18. Volpe MS, Adams AB, Amato MB, Marini JJ: Ventilation patterns influence airway secretion movement. Respir Care 2008, 53:1287-1294.

19. Rothen HU, Neumann P, Berglund JE, Valtysson J, Magnusson A, Hedenstierna G: Dynamics of re-expansion of atelectasis during general anaesthesia. Br J Anaesth 1999, 82:551-556.

20. Barker $\mathrm{M}$, Adams $\mathrm{S}$ : An evaluation of a single chest physiotherapy treatment on mechanically ventilated patients with acute lung injury. Physiother Res Int 2002, 7:157-169.

21. Blattner C, Guaragna JC, Saadi E: Oxygenation and static compliance is improved immediately after early manual hyperinflation following myocardial revascularisation: a randomised controlled trial. Aust $J$ Physiother 2008, 54:173-178.

22. Patman $S$, Jenkins $S$, Stiller $K$ : Manual hyperinflation: effects on respiratory parameters. Physiother Res Int 2000, 5:157-171.

23. Patman S, Jenkins S, Stiller K: Physiotherapy does not prevent, or hasten recovery from, ventilator-associated pneumonia in patients with acquired brain injury. Intensive Care Med 2009, 35:258-265.

24. Paulus F, Veelo DP, de Nijs SB, Beenen LF, Bresser P, de Mol BA, Binnekade JM, Schultz MJ: Manual hyperinflation partly prevents reductions of functional residual capacity in cardiac surgical patients: a randomized controlled trial 6. Crit Care 2011, 15:R187.

25. Choi JS, Jones AY: Effects of manual hyperinflation and suctioning in respiratory mechanics in mechanically ventilated patients with ventilator-associated pneumonia. Aust J Physiother 2005, 51:25-30.

26. Hodgson C, Denehy L, Ntoumenopoulos G, Santamaria J, Carroll S: An investigation of the early effects of manual lung hyperinflation in critically ill patients. Anaesth Intensive Care 2000, 28:255-261.

27. Dennis $D$, Jacob W, Budgeon C: Ventilator versus manual hyperinflation in clearing sputum in ventilated intensive care unit patients 4 . Anaesth Intensive Care 2012, 40:142-149.
28. Ahmed F, Shafeeq AM, Moiz JA, Geelani MA: Comparison of effects of manual versus ventilator hyperinflation on respiratory compliance and arterial blood gases in patients undergoing mitral valve replacement. Heart Lung 2010, 39:437-443.

29. Berney $S$, Denehy $L$ : A comparison of the effects of manual and ventilator hyperinflation on static lung compliance and sputum production in intubated and ventilated intensive care patients. Physiother Res Int 2002, 7:100-108.

30. Savian C, Paratz J, Davies A: Comparison of the effectiveness of manual and ventilator hyperinflation at different levels of positive endexpiratory pressure in artificially ventilated and intubated intensive care patients. Heart Lung 2006, 35:334-341.

31. Hodgson C, Ntoumenopoulos G, Dawson H, Paratz J: The Mapleson C circuit clears more secretions than the Laerdal circuit during manual hyperinflation in mechanically ventilated patients: a randomised crossover trial. Aust J Physiother 2007, 53:33-38.

32. Jellema WT, Groeneveld AB, van GJ, Wesseling KH, Westerhof N, Lubbers MJ, Kesecioglu J, van Lieshout JJ: Hemodynamic effects of intermittent manual lung hyperinflation in patients with septic shock. Heart Lung 2000, 29:356-366.

33. Paratz J, Lipman J, McAuliffe M: Effect of manual hyperinflation on hemodynamics, gas exchange, and respiratory mechanics in ventilated patients. J Intensive Care Med 2002, 17:317-324.

34. Paulus F, Binnekade JM, Vermeulen M, Vroom MB, Schultz MJ: Manual hyperinflation is associated with a low rate of adverse events when performed by experienced and trained nurses in stable critically ill patients: a prospective observational study. Minerva Anestesiol 2010, 76:1036-1042.

35. Patman S, Jenkins S, Bostock S, Edin S: Cardiovascular responses to manual hyperinflation in post-operative coronary artery surgery patients. Physiother Theory Pract 1998, 14:5-12.

36. Patman S, Jenkins S, Smith K: Manual hyperinflation: consistency and modification of the technique by physiotherapists. Physiother Res Int 2001, 6:106-117.

37. Paulus F, Binnekade JM, Middelhoek P, Vroom MB, Schultz MJ: Performance of manual hyperinflation: a skills lab study among trained intensive care unit nurses. Med Sci Monit 2009, 15:CR418-CR422.

doi:10.1186/cc11457

Cite this article as: Paulus et al: Benefits and risks of manual

hyperinflation in intubated and mechanically ventilated intensive care unit patients: a systematic review. Critical Care 2012 16:R145.

\section{Submit your next manuscript to BioMed Central and take full advantage of:}

- Convenient online submission

- Thorough peer review

- No space constraints or color figure charges

- Immediate publication on acceptance

- Inclusion in PubMed, CAS, Scopus and Google Scholar

- Research which is freely available for redistribution

Submit your manuscript at www.biomedcentral.com/submit
C Biomed Central 